\title{
Las Exposiciones Universales y Regionales como recurso didáctico en las Escuelas de Artes y Oficios (1886-1939)
}

\section{Universal and local Exhibitions as a didactic resource at the Arts and Trade Schools (1886-1939)}

\author{
M. Dolores DURÁN RODRÍGUEZ \\ Doctora en Ciencias de la Educación, Vigo
}

\begin{abstract}
RESUMEN: Este trabajo analiza la manera en que las exposiciones universales y regionales fueron utilizadas como recurso didáctico en las Escuelas de Artes y Oficios españolas, en el periodo 1886-1939, señalando los objetivos educativos y las actividades encaminadas a conseguirlos, así como la importancia de estas muestras en el desarrollo de las artes e industrias de ese periodo.
\end{abstract}

PALABRAS CLAVE: exposiciones universales y regionales, Escuelas de Artes y Oficios, excursiones, objetivos, arte, industria, ciencia, recursos didácticos, actividades.

ABSTRACT: This paper examines the way universal and local exhibitions were taken as a didactic resource at the Arts and Crafts Schools in the period 1886-1939, pointing out the educational objectives and the activities to fulfil them, as well as the importance of those exhibitions for the development of art and industry during those years.

KEY WORDS: universal and local exhibitions, Arts and Crafts Schools trips, objectives, art, industry, science, didactic resources, activities.

Como es sabido, en el movimiento renovador e innovador de la llustración - de cuyo espíritu son hijas las Escuelas de Artes y Oficios - las ciencias y las artes ocupan un lugar preponderante. Los enciclopedistas pertenecieron al sector activo que elaboró un nuevo orden económico y social, concediendo un alto valor a las ciencias, las artes y los oficios; y los hombres de la Revolución se interesaron por las Ciencias y las Bellas Artes, así como la transmisión de los oficios, organizando en París, en 1794, el Conservatorio de Artes y Oficios para la enseñanza de las artes y las ciencias aplicadas. En España, por su parte, Campomanes sostenía que todas las artes y oficios debían beneficiarse del progreso de la ciencia'. Este ilustrado tenía una visión clara del cambio trascendental que se estaba

1 Pedro R. de Campomanes: Discurso sobre la educación popular, Edición preparada por F. Aguilar Piñal, Madrid, Editora Nacional, 1978, p. 10. Es importante recordar que la prioridad que los ilustrados conceden a la cuestión educativa está íntimamente ligada a su proyecto más evidente: la reforma económica del país, como señalan 
operando en Europa, con unas técnicas más racionales y científicas, de ahí que abogase por una educación en los nuevos métodos y por el fomento de la industria y el comercio².

Los escaparates de la ciencia fueron las exposiciones universales, especialmente en la segunda mitad del siglo XIX y primeros años del XX, por lo que no es sorprendente el interés que las Escuelas de Artes y Oficios - centros de educación técnica popular - experimentaron hacia ellas. Estas magnas exhibiciones, en todas sus variedades, se convirtieron para estas Escuelas en uno más de sus recursos didácticos y un refuerzo de la enseñanza. Pero no sólo eso. También fueron a menudo un medio para exponer los resultados prácticos de las actividades de sus alumnos y una excelente forma de entrar en conocimiento de las novedades científicas, industriales y artísticas que habrían de incorporarse al currículo escolar, así como de novedades en materia pedagógica.

Las exposiciones universales mostraban los logros industriales, científicos, educativos y artísticos de los países participantes, mientras que las regionales o locales se referían a zonas o regiones concretas. Como sabemos, las Escuelas de Artes y Oficios no eran ajenas a las industrias de la comarca en que se hallaban; bien al contrario, estas industrias conformaban, hasta cierto punto, sus planes de estudios, e incluso propiciaban su propia existencia. Pues en no pocos casos la creación de un Escuela de Artes y Oficios en España se debió a la necesidad de desarrollar o perfeccionar la industria o industrias locales ${ }^{3}$. Hay, pues, una relación inmediata entre estos centros y los certámenes industriales regionales. Por otro lado, las muestras artísticas servían a nuestras Escuelas para conocer las nuevas tendencias del arte y, en el caso de las regionales, para mostrar las obras de profesores y alumnos, amén de propiciar el estudio de las obras de arte.

Pero, además de todo ello, las exposiciones, especialmente cuando para acudir a ellas se hacían excursiones escolares, representaban todo un abanico de posibilidades didácticas que las Escuelas aprovecharon para reforzar o completar la enseñanza, como se verá a continuación.

\section{Las Escuelas de Artes y Oficios: definición, objetivos y clases}

Según sabemos, las Escuelas de Artes y Oficios eran, en la época que estudiamos, unas instituciones educativas dedicadas a la enseñanza de las artes aplicadas y de los oficios artísticos que no constituían propiamente un nivel o ciclo del sistema educativo general, sino más bien un tipo de formación profesional que preparaba para el desempeño

R. Flecha, F. López y R. Saco: Todos los ilustrados insistieron infatigablemente en el tema de la instrucción pública, no como gesto generoso hacia el pueblo iletrado, sino porque en su propagación radicaba, en buena medida, el éxito de la reforma que habían resuelto acometer. Consecuentemente, abogaron por una educación elemental común a todos los ciudadanos, haciendo hincapié, de modo particular, en los saberes técnicos, en cuyo abandono veían una de las principales causas de la decadencia (Ramón Flecha, Fernando López y Raquel Saco: Dos siglos de educación de adultos. De las Sociedades de Amigos del País a los modelos actuales, Barcelona, Roure, 1988, p. 17)

2 Pedro R. de Campomanes, op. cit., p. 11.

3 Véase M. Dolores Durán Rodríguez: La Escuela de Artes y Oficios de Vigo durante el primer tercio del siglo XX. El centro que contribuyó a crear el gran Vigo, Vigo, Ediciones Cardeñoso, 2008. 
de actividades artesanales y artísticas. Su objetivo primordial consistía en la formación técnica del obrero o artesano, y/o el perfeccionamiento de un oficio. Pero podían tener también otros objetivos, según las necesidades de la región en que se asentaban: completar una educación primaria deficiente, la mejora de las condiciones económicas y sociales de los obreros, y fomentar en ellos el gusto por el estudio. En algunos centros de Galicia se añadían dos objetivos más, que parecen reflejar las ansias de la población de finales del siglo XIX: despertar en los gallegos el espíritu de empresa y lograr la igualdad de clases en la faceta cultural ${ }^{4}$.

Estos centros de educación técnica de adultos surgieron en España a partir de mediados del siglo XIX (en Galicia, a finales de ese siglo) y, aunque tuvieron su origen en los franceses, aquí tienen características propias. Su creación se debe, bien a la iniciativa oficial (las llamadas Escuelas de Artes y Oficios oficiales o de distrito), bien a iniciativa privada (promovidas por sociedades privadas, por municipios, por diputaciones, por Sociedades Económicas de Amigos del país...), pero para el tema que nos ocupa estas diferencias son irrelevantes. En Galicia tenemos ejemplos de todas ellas: debida a iniciativa estatal, la de Santiago de Compostela; por iniciativa de una sociedad privada de artesanos, la de Vigo; fundadas por las diputaciones, la de Ferrol y la de Ourense; debida a la Sociedad Económica de Amigos del País de la ciudad, la de Pontevedra, etc.

\section{Las excursiones escolares}

Al analizar las relaciones entre las exposiciones universales y regionales y las Escuelas de Artes y Oficios, debemos comenzar por hablar de las excursiones escolares, pues la acción educativa de dichas muestras va ligada, a menudo, a esos viajes organizados de profesores y alumnos para verlas y estudiarlas. De la acción conjunta de ambas surgirán las numerosas actividades didácticas a que hemos aludido.

Si tomamos como ejemplo la Escuela de Artes y Oficios de Vigo en las primeras décadas del pasado siglo, podemos asegurar que en ella se dieron múltiples excursiones escolares, habiendo constancia documental de que estas actividades se preparaban de antemano, detenidamente, conforme a unos fines y contenidos, y siguiendo un modelo previamente programado. En casi todas estas excursiones, a diferencia de los "paseos" o "visitas" cortas, se asistía a una exposición, entre otras actividades.

Las excursiones, reflejo de la influencia pedagógica de la Institución Libre de Enseñan$z^{5}$ y de la llamada Escuela Nueva ${ }^{6}$, estaban previstas en la legislación, desde la Primaria

4 Reglamento de la Escuela de Artes y Oficios de Vigo. 1886. Madrid, Imp. Fortanet, 1886, p. 4

5 Pese a la vocación netamente universitaria de la Institución Libre de Enseñanza, ésta mostró desde sus propios estatutos un gran interés por la cultura popular, y en sus casi 60 años de existencia, en su Boletín aparecieron más de un centenar de artículos sobre las escuelas de adultos, la educación de la mujer, la educación del obrero, etc. (Ramón Flecha, Fernando López y Raquel Saco, op. cit., p. 40).

6 La educación nueva es la educación que aspira a formar la individualidad vital humana dentro de la colectividad, en un ambiente de libertad, por medio de la actividad nos define Lorenzo Luzuriaga a título provisional, el cual señala como ideas esenciales y básicas de la misma las de actividad, vitalidad, libertad, individualidad 
(R. O. 10 de abril de 1918 que da facilidades a los maestros para la práctica de este procedimiento de enseñanza ${ }^{7}$ ), hasta la propia educación de adultos en las Escuela de Artes y Oficios, aunque en este caso sólo referida a visitas prácticas (R. D. 5 de noviembre de 1886, que establece en su artículo $3^{\circ}$ que la enseñanza en estos centros incluirá visitas hechas por los alumno a fábricas o talleres, bajo la dirección de sus respectivos Profesores o Maestros de taller $\left.{ }^{8}\right)$.

En la Escuela de Artes y Oficios de Vigo, que a comienzos de la pasada centuria contaba con un director institucionista, Fernando García Arenal, que destacaba en sus escritos este tipo de actividades, se contaba con un presupuesto anual destinado a excursiones, como ocurría en otros centros similares. Pero el aspecto financiero, en este como en otros aspectos de la enseñanza en las Escuelas de Artes y Oficios, constituía siempre un problema, siendo reducido el número de escolares que podían beneficiarse de las excursiones. Así se recoge en documentos de archivo del citado centro vigués, o bien de la Escuela de Artes y Oficios de A Coruña, en fecha tan tardía como 1932, cuando en esta última se habla de la "excursión instructiva escolar y voluntaria de alumnos y profesores", celebrada al final del curso: Fue esta una excursión plena de utilidad cultural, y creemos que los organismos llamados a ello deben fomentar estas excursiones, donando cantidades para que aquellos alumnos que carezcan de medios puedan también recoger los beneficios que se alcanzan de esta suerte 9 .

Las excursiones son, pues, parte de la nueva pedagogía, cuyas bases las sientan las concepciones educativas formuladas por Rousseau en el Emilio, defendiendo un mayor contacto con la naturaleza, y las propuestas de Pestalozzi, que elevó a categoría científica la intuición, una metodología basada en la observación directa de la naturaleza, como estímulo para la educación integral del niño ${ }^{10}$. Así como los métodos intuitivos de Froëbel de la educación integral. España incorporó estos planteamientos educativos tardíamente, en el siglo XIX: La enseñanza-aprendizaje fuera del aula, es decir, la práctica de salidas y excursiones del maestro con sus alumnos a un determinado centro, lugar o paraje en donde éstos reciben información y explicaciones de interés instructivo-educativo que se refieren al mismo y en cuyo desarrollo se utilizan diferentes medios de locomoción, es una actividad relativamente reciente en España que se inicia con lentitud hace poco más de un

y colectividad; sin olvidar que los antecedentes históricos de este movimiento renovador, que es un fenómeno común a todas las épocas y a todos los pueblos, se hallan, partiendo de las ideas y los métodos pedagógicos innovadores del mundo clásico (Sócrates y Platón frente a la educación tradicional griega y las ideas innovadoras de Cicerón y Quintiliano respecto a la educación romana), y pasando por las concepciones de los pedagogos humanistas del Renacimiento, en las ideas revolucionarias de Rousseau y Pestalozzi frente a la educación de su tiempo, y las concepciones integradoras del siglo XX frente al intelectualismo del XIX (Lorenzo Luzuriaga: La nueva educación, Buenos Aires, Losada, S. A., 1967, pp. 15 a 30).

7 Alcubilla (dir. Marcelo Martínez-Alcubilla): Boletín Jurídico-Administrativo. Anuario de Legislación y Jurisprudencia. Apéndices al Diccionario de la Administración Española. Apéndice 1918, Madrid, Administración: Augusto Figueroa, 1918, p. 118.

8 Gaceta de Madrid, nº 310, de 6-11-1886, "Real Decreto del Ministerio de Fomento de 5-11-1886", art. 3º, p. 378. 9 Memoria reglamentaria del curso 1931-32. Escuela de Artes y Oficios de La Coruña, pp. 4-5.

10 María Luisa Gutiérrez: "Las excursiones escolares y la interdisciplinariedad en ciencias sociales: Alejandro de Tudela”, en Iber. Didáctica de las Ciencias Sociales, Geografía e Historia, 2001, pp. 113-120. 
siglo ${ }^{11}$. Como nos recuerda el profesor Ángel Serafín Porto Ucha, la metodología de Pestalozzi y su seguidor Froëbel, está presente en la Institución Libre de Enseñanza, y significa la adopción del método intuitivo, mediante la libre participación del alumno (la actividad) y una nueva consideración del espacio escolar ${ }^{12}$.

La práctica de los viajes escolares fue introducida en España por la Institución Libre de Enseñanza, que hizo de las excursiones una de las claves distintivas de la educación geográfica. Es de conocimiento común que, dentro del horizonte educativo de la ILE, la educación geográfica ocupó un lugar destacado. Se procuró poner en práctica una enseñanza geográfica moderna, acorde con los puntos de vista más actualizados de la Europa de su tiempo, empeñada, como decía Torres Campos $^{13}$, en desarrollar el espíritu de observación de los alumnos y ayudarles así a hacer la geografía por sí mismos. Para lograr esa educación geográfica de carácter intuitivo y realista, y apoyada en la visión personal de las cosas, era necesario que el alumno se acercase a la realidad, que se pusiese en contacto con el paisaje. Por eso eran fundamentales las excursiones ${ }^{14}$. Torres Campos, durante el VI Congreso Internacional de Ciencias Geográficas que se desarrolló en Londres en 1895, afirmó refiriéndose a ellas: Son un excelente medio, porque ensanchan las ideas y favorecen el desarrollo del espíritu de observación de los alumnos, sirviendo para que éstos hagan la geografía por sí mismos. En los libros se encuentra la geografía formada; en los viajes se la elabora ${ }^{15}$.

La modernización de la enseñanza de la geografía promovida en la Institución por Torres Campos, se apoyaba, ante todo, en la concepción naturalista del conocimiento geográfico, en la idea de que es en el orden natural, en el conjunto de factores y relaciones naturales, donde hay que buscar las claves para lograr entender correctamente los hechos geográficos, incluidos los humanos ${ }^{16}$. La importancia que la ILE concedía a la enseñanza geográfica queda patente en los relatos de excursiones de la Institución que fueron apareciendo en su Boletín y en el "cuestionario de excursiones" que elaboró Cossío para orientar a los alumnos en sus salidas y observaciones.

\footnotetext{
11 Ibidem

12 Ángel Serafín Porto Ucha: La Institución Libre de Enseñanza y la renovación pedagógica en Galicia, Sada - A Coruña, Ediciós do Castro, 2005, p. 144.

13 Rafael Torres Campos (1853-1904), abogado y militar, fue el primer y principal impulsor de la geografía moderna en la ILE, con un protagonismo destacado en la labor de renovación de la enseñanza geográfica impulsada por el núcleo institucionista. La propia institución le envió a la Exposición Universal de París de 1878, lo que le permitió conocer de forma directa las novedades en el campo de la enseñanza geográfica que allí se presentaron; una de estas novedades, que Torres Campos incorporó con especial fortuna al funcionamiento educativo de la Institución, fue precisamente, como se recordaría después en las páginas de su Boletín, "el sistema de excursiones escolares" ("Francisco Giner de los Ríos. Datos biográficos", Boletín de la Institución Libre de Enseñanza, XXXIX, 659-660 (1915), p. 36, recogido en Nicolás Ortega Cantero: "Educación geográfica y valoración del paisaje en la Institución Libre de Enseñanza”, en Boletín de la Institución Libre de Enseñanza 2004, (55), 42-68)

14 Nicolás Ortega Cantero, op. cit.

15 Ibidem.

16 Ibidem
} 


\section{Las exposiciones universales, regionales, anuales del centro, y otros certámenes}

Es necesario, ante todo, distinguir las variedades de exposiciones con respecto a las Escuelas de Artes y Oficios. Están, en primer lugar, las exposiciones anuales de los trabajos que los alumnos realizaban durante el curso escolar. Estas se celebraban generalmente al final del curso en una estancia del centro y abiertas al público, y eran preceptivas para todas las Escuelas oficiales y también para las privadas que las incluían en sus reglamentos. Están previstas desde un primer momento, pues ya en el citado R. D. de 5 de noviembre de 1886, que organiza este tipo de centros por primera vez en España, creando además siete Escuelas de distrito, se alude a ellas en el artículo 22: Todos los trabajos premiados serán expuestos al público y pertenecerán a la Escuela... ${ }^{17}$ Los trabajos pasaban después a formar parte del Museo de la Escuela. Estas exposiciones anuales representaban un refuerzo positivo de la enseñanza, pues servían de acicate a los alumnos, y también significaban un examen a los docentes por parte de la dirección del centro, así como un control a este mismo por parte de las instituciones que los mantenían. Eran también un medio para aumentar el prestigio de la Escuela ante las familias de los alumnos y ante el público en general.

Tenemos luego las exposiciones a nivel general, de todo el país, a las que las Escuelas oficiales deben concurrir preceptivamente, tal como se establece en el R. D. de 20 de agosto de 1895:

Con el propósito de estimular a los artesanos para que se instruyan en las aplicaciones del arte a la industria, se organizarán cada dos años Exposiciones artístico-industriales, que alternen con las de Bellas Artes. Las obras premiadas en las Exposiciones industriales constituirán un Museo, donde podrán estudiarse los trabajos que, por su belleza y feliz adaptación a las necesidades de la vida, satisfagan las exigencias del progreso de las artes industriales $^{18}$.

Están también las exposiciones regionales, locales, y nacionales, entre las que destacan, en Galicia, las celebradas en Santiago de Compostela en 1858 (la primera de Galicia), en 1875 y en $1909^{19}$, así como el Certamen de Artes y Oficios celebrado en Pontevedra en $1880^{20}$. Aquí incluiríamos también al II Certamen Regional del Trabajo de Galicia, celebrado en Ferrol en 1935. Y tenemos por último las exposiciones universales, de carácter general y con la concurrencia de numerosos países.

17 Gaceta de Madrid no 310 ... op. cit., p. 378.

18 Real Decreto y Reglamento Orgánico de las Escuelas de Artes y Oficios de 20 de Agosto de 1895, Madrid, J. Fernández, impresor, Tahona de las Descalzas, 6, dup., 1895, p. 7.

19 Margarita Barral Martínez: "O espírito expositivo na época contemporánea e a organización e desenvolvemento da Exposición Regional Gallega de 1909, en Exposición Gallega de 1909. Santiago. Conmemoración do 1ำ Centenario "Exposición Regional Gallega Santiago 1909, Consorcio de Santiago, Museo do Pobo Galego, Instituto de Estudios Galegos "Padre Sarmiento", MMX, pp.121 a 194.

20 Diputación de Pontevedra / Museo de Pontevedra: Os pensionados da Deputación de Pontevedra (18641933), 2003, p. 146. 
Las exposiciones universales, en palabras de Joaquín Yarza Luaces, son acontecimientos concebidos como escaparate público para dar a conocer los adelantos de la industria, el comercio y las artes ${ }^{21}$. Tuvieron como punto de referencia original diversas muestras que, con ese objetivo pero de ámbito estrictamente nacional, venían celebrándose en algunos países. A partir de 1851 se internacionalizaron, tomando el adjetivo de universales, y se institucionalizaron como un acontecimiento periódico que se viene prolongando hasta nuestros días ${ }^{22}$. Mariano Carderera, que estudió detenidamente la Exposición Universal de Londres de 1862, señala el punto de partida de estas magnas exhibiciones industriales a mediados del siglo XVIII: Desde mediados del siglo último, época en que ya se dejaba ver su necesidad y su porvenir, fue llamada la industria a ostentar sus progresos y desde entonces principian las exposiciones industriales ${ }^{23}$.

Las exposiciones se convirtieron en una parte fundamental del engranaje de la sociedad industrial, cumpliendo la función de mediación psicológica que permitía a la población aclimatarse a la industrialización de la sociedad, convirtiéndose el espectador en protagonista fundamental del crecimiento de las naciones occidentales ${ }^{24}$. La Great Exhibition of the Works of Industry of all Nations, como se llamaba la de Londres de 1851, es considerada como la primera Exposición Universal, e inaugura un fenómeno social de trascendentes consecuencias para la cultura occidenta ${ }^{25}$. Como ejemplo de lo que era una muestra de estas características en el siglo XIX, transcribimos la descripción que de la Exposición Universal de Londres de 1862 nos ofrece el citado Carderera:

Dividido con desigualdad notable aquel inmenso espacio entre las diversas naciones que han concurrido a la solemnidad, cada una de ellas ostenta en su especial departamento los productos de su suelo con los de su inteligencia y laboriosidad, presentando el conjunto muestras inequívocas de todos los grados de cultura y de civilización. El cuadro que se ofrece a la vista desde cualquiera de las plataformas, de donde se descubre toda la nave principal y uno de los cruceros, es de lo más grandioso y deslumbrador que jamás he visto. Aquella multitud de objetos agrupados de la manera más agradable bajo de elegantes arcos, entre banderas y trofeos de variedad suma y entre estatuas y otras obras del arte, aquella diversidad de trages (sic) de los visitadores y aquel murmullo producido a la vez por el sonido de diferentes instrumentos músicos, por el agua que arrojan los saltadores, por el estruendo de las máquinas en movimiento, por las palabras pronunciadas con el acento de mil idiomas diferentes, dejan absorto al espectador sin que pueda darse cuenta de lo que tiene ante los ojos... ${ }^{26 .}$

$21 \mathrm{http}: / /$ www.artehistoria.jcyl.es/arte/contextos/5147.htm

22 Ibídem

23 Mariano Carderera: La Pedagogía en la Exposición Universal de Londres de 1862, Madrid, Imprenta de D. Victoriano Hernando, c/ Arenal, 11, 1863, pp. 2-3.

24 Daniel Canogar: Ciudades efímeras. Exposiciones Universales: Espectáculo y Tecnología, Madrid, Julio Ollero Editor Imaginario, 1992, pp. 21-23.

25 Ibidem

26 Mariano Carderera, op. cit., pp. 7-8. 
Durante casi dos siglos, las exposiciones universales han contribuido al desarrollo de la humanidad gracias a que, además de ser un especio de diálogo internacional, son el medio a través del cual se difunde la riqueza cultural de los pueblos y se dan a conocer las últimas tendencias del mundo ${ }^{27}$. Algunas de las más sobresalientes del siglo XX, hasta 1939, fueron las de París 1900, San Louis Missouri 1904, San Francisco 1915, Barcelona 1929, Chicago 1933, Bruselas 1935, París 1937, y Nueva York $1939^{28}$.

Muy pronto se vio la relación de estas muestras, no sólo con el desarrollo industrial, comercial, y artístico, sino también con la enseñanza técnica popular. La enseñanza de las exposiciones alcanza a todos, declara Carderera, señalando a continuación algunas de esas enseñanzas para el artesano: Un carpintero, un tejedor, otro cualquier artesano, con lo que más puede interesarle en su profesión, encuentra allí visitadores de todos los países que, proponiéndose igual estudio, entrarán con gusto en explicaciones provechosas para unos y otros (...) Entre los útiles y herramientas algunas serán acaso desconocidas para él (...) Verá por lo menos las mejoras o modificaciones en ellas introducidas en los países más adelantados en particular ${ }^{29}$.

Todo comienza con la invención y la información: de este doble producto de la Revolución Industrial nacieron las exposiciones universales en el siglo XIX, según sostienen Aimone y Olmo en un trabajo sobre estas muestras ${ }^{30}$. Por su parte, $M^{-}$del Mar del Pozo Andrés señala que las exposiciones universales o mundiales, celebradas profusamente en la segunda mitad de dicho siglo, pueden considerarse como muestras interdisciplinarias internacionales, ilustrativas del progreso industrial y social alcanzado por los países occidentales, y demostrativas del modus vivendi existente en los pueblos africanos y orientales, que aún mantenían su exotismo y misterio para los hombres europeos ${ }^{31}$. Según esta autora, el objetivo fundamental de estas muestras, llenas de espectacularidad y colorido, era la exhibición didáctica de los logros técnicos conseguidos en multitud de manifestaciones particulares: Economía, Ciencias Naturales, Etnología, Comercio, Comunicaciones, Industria, Educación y Artes Plásticas. En este sentido, constituyen un elemento básico para el análisis de la cultura y la sociedad decimonónica ${ }^{32}$. Estas características también se pueden aplicar a las exposiciones celebradas en las primeras décadas del siglo XX.

El tema de la educación comenzó a adquirir gradual importancia en las exposiciones universales a partir de la tercera de ellas, la ya citada de Londres de 1862. En ella se consagró una clase para "Métodos y material de enseñanza"; en París (1867), fueron dos las clases reservadas ("Métodos y material de enseñanza para los niños" e "Instituciones de cultura popular"); en Viena (1873), nos encontramos ya con un grupo entero - el XXVI - de carácter pedagógico, en el que, bajo el título de "Educación, Enseñanza, Instrucción", se

27 http://www.expo2010mexico.com.mx/es/PM_historia.htm

28 Ibídem

29 Mariano Carderera, op. cit., pp. 16-17.

30 Linda Aimone y Carlo Olmo: Les Expositions universelles, 1851-1900, París, Belin, 1993.

31 María del Mar del Pozo Andrés: "Presencia de la pedagogía española en las Exposiciones Universales del XIX", en Historia de la Educación, 2, (1983), pp. 165-172

32 lbidem. 
incluía: tipos y modelos de construcciones escolares; muebles y material para las escuelas primarias; obras y publicaciones periódicas sobre temas educativos; métodos y estrategias didácticas; historia, organización, reglamento y estadística estudiantil de instituciones elementales, secundarias y Universidades; Colegios de sordo-mudos, ciegos y retrasados; Escuelas Normales, Industriales y Profesionales, de Artes y Oficios y Comerciales; formación de adultos e instrucción popular. Este esquema temático perduró para las restantes Exposiciones, agrupándose las diversas producciones pedagógicas de acuerdo con estas secciones ${ }^{33}$.

La atención de las Escuelas de Artes y Oficios hacia estos eventos fue, por lo tanto, una consecuencia de todo ello. De hecho, estas exhibiciones serían fundamentales para el desarrollo y divulgación de este tipo de centros en ciertos lugares, como el Reino Unido, como señala el ministro de Fomento de España, Carlos Navarro y Rodrigo, en la parte expositiva del mencionado R. D. de 5 de noviembre de 1886:

La Exposición universal de 1851 hizo conocer a la Gran Bretaña que para competir con las industrias extranjeras tenía necesidad imperiosa de difundir en sus clases populares la educación artística de que carecía... ${ }^{34}$

Y esa educación artística se concretaba en la enseñanza del Dibujo, de la que carecían las clases populares británicas. Visto el problema, se trató de darle solución:

Reconociendo desde luego que el mal estaba en la falta de instrucción en el dibujo, reservado hasta entonces a las clases acomodadas, se concibió la idea de popularizarlo y se emprendió la obra con ese talento práctico y esa persistencia que caracteriza a los ingleses, creando un centro directivo con objeto de desarrollar el gusto artístico entre los obreros, estableciendo una Escuela Normal, formando profesores, preparando colecciones de modelos de todas clases y dimensiones, y abriendo cursos de dibujo en la mayor parte de las escuelas populares de las ciudades del reino ${ }^{35}$.

Así pues, Londres contó, en los años siguientes, con una Escuela Normal de ciencias y artes a la que se agregaron clases para los obreros, donde se les enseñó a estos la arquitectura, el trabajo en metales, la mecánica, el bordado, el encaje, el grabado en madera, la pintura en porcelana, y todas las artes aplicadas ${ }^{36}$.

La relación entre las Escuelas de Artes y Oficios españolas y las exposiciones universales y regionales, fue una constante en todo este periodo: los centros enviaban a ellas a sus escolares, participaban en ellas como expositores con trabajos de alumnos y profesores, y las tomaban como referencia industrial, comercial y artística. El haber obtenido galardón en estas muestras era un mérito para la docencia en ciertos momentos. Por ejemplo, en el decreto de 27 de mayo de 1936 del Ministerio de Instrucción Pública sobre las Escuelas de Artes y Oficios, leemos en el artículo $1^{\circ}$ 
Para desempeñar plazas de Profesores de término, Auxiliares y Ayudantes meritorios que pertenezcan a la Sección artística de las Escuelas de Artes y Oficios será preciso reunir alguna de las condiciones siguientes: Poseer el título de Profesor de Dibujo que expiden las Escuelas Superiores de Pintura, Escultura y Grabado, o haber sido premiado con medalla de cualquier clase en Exposiciones nacionales organizadas por el Ministerio de Instrucción pública, o en las Internacionales de igual naturaleza, o haber sido pensionado por oposición en la Academia Española de Bellas Artes en Roma, cumpliendo el plazo y las condiciones reglamentarias de la pensión, o haber obtenido con iguales requisitos las pensiones Piquer o Conde de Cartagena ${ }^{37}$.

Igualmente, referido este a vacantes de Maestros y Ayudantes de Taller de los mismos centros, el decreto de 28 de marzo de ese año establece como condición segunda para la provisión de esas plazas en concurso-oposición, mejor calidad y, dentro de ella, mayor cantidad de Medallas obtenidas en Exposiciones nacionales e internacionales; y, como condición tercera, mayor número de premios y accésit obtenidos en tales Exposiciones y otros concursos ${ }^{38}$.

En lo que respecta al beneficio que los alumnos podrían obtener de las exposiciones, el interés oficial por las mismas viene de lejos. El ministerio de Agricultura, Industria, Comercio y Obras Públicas, en R. O. de 23 de mayo de 1900, dispone que las Escuelas de Artes y Oficios envíen a París, por cuenta del Estado, a dos alumnos, para que estos escolares estudien en la exposición universal que allí se va a celebrar ese año ${ }^{39}$. Los propios escolares eran conscientes de la importancia que para su formación laboral tenían estas exposiciones, como lo demuestra la solicitud que, antes de que el Ministerio hubiese decidido dichas subvenciones, 15 alumnos de la Escuela de Artes y Oficios de Vigo habían dirigido a la dirección para que el centro les llevase a esa misma exposición. En efecto, el $1^{\circ}$ de febrero de 1900, estos escolares se dirigieron al Director y al Claustro de profesores de la Escuela en los siguientes términos:

Los que suscriben, alumnos matriculados desde la fundación de la Escuela, respetuosamente exponen: que con motivo de celebrarse este año la Exposición Universal de París, y viendo el atraso en que los obreros de esta ciudad nos encontramos con relación a los de Madrid, Barcelona, Bilbao, Sevilla, y a los de los demás países extranjeros a quienes se debe parte de su adelanto a lo que han estudiado y visto en las anteriores Exposiciones subvencionadas por las Corporaciones Oficiales, verían con gusto a más de quedarles altamente reconocidos, que por los medios que estén a su alcance se digne prestarnos su poderoso concurso, a fin de conseguir que puedan asistir a la próxima Exposición de París los alumnos que se dignen nombrar al efecto ${ }^{40}$.

37 Aranzadi, Estanislao de (dirección y administración): Repertorio cronológico de Legislación 1936, 1ª edición 6 y 6 bis, Pamplona, p. 513.

38 Ibídem, p. 310.

39 Gaceta de Madrid de 24-5-1900, Real Orden 23 de mayo de 1900. También están las Reales Órdenes de 23 de junio y 11 de julio de 1900 sobre el mismo tema. Esta R. O. se refería a las Escuelas de Artes y Oficios oficiales y también las privadas con programa oficial, caso de la Escuela de Artes y Oficios de Vigo, que solicitó y obtuvo esas pensiones.

40 Archivo de la Escuela de Artes y Oficios de Vigo. "Instancia de 15 alumnos de la Escuela de Artes y Oficios de Vigo, 1-2-1900", Carpeta de Excursiones. 
Como vemos, los argumentos que esgrimían iban en la línea de lo que se admitía generalmente en esa época: el atraso de los obreros se paliaría con la asistencia a las exposiciones. Es conveniente señalar, como recuerda el profesor Porto Ucha, que tanto el desarrollo de la enseñanza primaria como el de la enseñanza técnica fue producto de la preocupación de la España liberal, y que este movimiento a favor de la enseñanza técnica se vio favorecido por el auge que alcanzaron las Exposiciones universales en la segunda mitad del siglo XIX ${ }^{41}$.

En cuanto a la enseñanza artística, y circunscribiéndonos a la región gallega, $X$. M. López Vázquez nos recuerda que es a finales del siglo XIX cuando el arte empieza a presentarse como un componente esencial en la definición de la cultura y, consecuentemente, como un magnífico indicador del desarrollo de la sociedad o del país. Y es ahí, como baremo del desarrollo de la sociedad, que el arte tendrá un lugar destacado en las Exposiciones Regionales que, cunha finalidade claramente propagandística, se celebran daquela en diversas cidades da nosa terra ${ }^{42}$.

\section{Participación de las Escuelas de Artes y Oficios en las Exposiciones}

Entre los numerosos ejemplos de participación de estos centros en las exposiciones universales y regionales, podemos citar los siguientes: en la Exposición de Bellas Artes de Madrid, de 1907, obtuvo una medalla la Escuela de Artes y Oficios de Oviedo ${ }^{43}$; en la Exposición Nacional de Arte Decorativo de 1923, fue galardonado con primera medalla el profesor de la Escuela de Artes y Oficios Artísticos de Toledo, Francisco Pérez Dolz ${ }^{44}$; y esta Escuela envía también trabajos de profesores y alumnos a la Exposición Ibero-Americana de Sevilla de 192945 . Por su parte, los destacados orfebres y esmaltadores estilo "art déco", Eloy y Osmundo Hernández Calzada, tras haber realizado sus estudios en la Escuela de Artes y Oficios de Valladolid, aportan obras suyas a la Exposición de Artes Gallego de 1924 y a la Exposición Internacional de Filadelfia ${ }^{46}$.

En el ámbito gallego, la Escuela de Artes y Oficios de Santiago, que en 1906 celebró una Exposición Artístico Industrial de trabajos de sus alumnos y ex alumnos, de gran calidad y éxito, recibiendo el Premio de Honor y donativo de Alfonso XIII, y siendo premiada su alumno Alfredo Fariña Iglesias; en 1911, este centro obtiene la segunda Medalla en la

41 A. S. Porto Ucha: "Las Escuelas de Artes y Oficios en Galicia y su contribución al desarrollo del mundo urbano (1886-1902)", en L'Educació al món urbá, IX Jornades d'Historia de l'educació als paisos catalans, Escola Universitaria del Professorat d'E.G.B. de la Universitat de Barcelona, 1987, pp. 345 a 355.

42 X. Manuel López Vázquez: "Introducción", en A. Pulido Novoa (dir.), Artistas Galegos Pintores (Novecentos), Vigo, Nova Galicia Edición, S. L., 1998, pp. 15 a 25.

43 Escuela de Artes y Oficios de Oviedo. Memoria reglamentaria de 1907-1908, p. 8.

44 Escuela de Artes y Oficios Artísticos de Toledo. Memoria de 1923-24, Toledo, Talleres Gráficos de Rafael G. Menor, 1924, p. 4.

45 Escuela de Artes y Oficios Artísticos de Toledo. Memoria de 1928, p. 9

46 Román Padín Otero: "Los hermanos Hernández. Una familia de orfebres y esmaltistas estilo art déco", en (http://www.elcorreogallego.es/vida-social/ecg/hermanos-hernandez-familia-orfebres-e...) 
Exposición Nacional de Artes Decorativas e Industrias Artísticas celebrada en Madrid ${ }^{47}$. En la Exposición de Bruselas de 1914, el alumno de la Escuela de Artes y Oficios de Vigo, José Cameselle Rial, consigue una medalla ${ }^{48}$. La Escuela de Santiago también consiguió medalla de oro en la Exposición Regional de Lugo de 1916, y Diploma de Honor en la Exposición Ibero-Americana de Sevilla de $1929^{49}$, donde Galicia tenía pabellón. Y el pintor Carlos Sobrino Buhigas, profesor de la Escuela de Artes y Oficios de Vigo, que obtuvo, entre otras, una medalla en la Exposición Iberoamericana de Sevilla de $1931^{50}$.

Las Escuelas de Artes y Oficios de Vigo y Santiago concurren, con pabellones propios, a la Exposición Regional Gallega del Año Santo de 1909 de la ciudad compostelana, destinada a demostrar el grado de adelanto de la industria, del comercio y de las artes: tratábase de exhibir ao público os novos artigos producidos tanto no exterior como, sobre todo, na propia Galicia, de ofrecer nun escaparate a súa capacidade produtiva, potencial ou real, e de mostrar o camiño polo que habería de transitar o futuro ${ }^{51}$. La Escuela de Santiago tomará parte muy activa en su preparación y organización por parte de algunos profesores, como Ramón Núñez, Enrique Mayer Castro, José Mª Fenollera, Rafael de la Torre, Álvaro Caula y Manuel H. Álvarez Reyero, así como el trabajo de los fotógrafos Enrique Sánchez Guerra y Manuel Chicharro, ambos ex alumnos de la propia Escuela ${ }^{52}$. Profesores y alumnos fueron premiados en la muestra, y la Escuela, como institución, obtuvo el Diploma de Honor ${ }^{53}$.

Las Escuelas de Artes y Oficios, como era de esperar, solían participar de modo especial en las exposiciones regionales que se celebraban en sus zonas respectivas, como estos casos de la Exposición Gallega de 1909, y el de la Escuela de Artes Aplicadas y Oficios Artísticos de Zaragoza, que debió su edificio a la Exposición Hispano-Francesa de 1908, conmemorativa del Centenario de los Sitios ${ }^{54}$. Podemos nombrar también los casos de las Escuelas de Sevilla y Málaga y su participación, con pabellón propio, en la citada Exposición Ibero-Americana de Sevilla de 1929. En la memoria del curso 1917-18 de la Escuela de Artes y Oficios de Sevilla se prevé, incluso, un cambio de ubicación de la misma gracias a ella, pues el Comité de dicha Exposición Hispano-Americana, que iba

47 José Sousa y Fernando Pereira: Historia de la Escuela de Artes y Oficios de Santiago, A Coruña, Editorial Diputación Provincial, 1988, p. 77.

48 G. González Martín: “Crónica Sentimental”, en Faro de Vigo de 25-6-1998. Este alumno es el fundador de "La Belga", la conocida empresa de elaboración de vidrio.

49 Memoria de la Escuela de Artes y Oficios Artísticos de Santiago de Compostela. Curso de 1947 a 1948 , Santiago, Imprenta Paredes, 1948, p. 23.

50 G. González Martín: Pasión por Vigo. Vida y obra del cronista Rodríguez Elías, Vigo, Instituto de Estudios Vigueses, 2005, p. 341.

51 Ángel I. Fernández González y Jesús Giráldez Rivero: "Santiago 1909: A economía galega no escaparate da Exposición Regional", en Exposición Gallega de 1909...., op. cit, pp. 79 a 119. Sin embargo, es de señalar que la industria de más auge en la Galicia de la época - la derivada del mar, en las Rías Bajas - no tuvo apenas representación en la misma, como nos recuerdan estos autores.

52 José Sousa y Fernando Pereira: op. cit., pp. 75-76.

53 Ibídem

54 Jaime Ángel Cañellas: La Escuela de Artes Aplicadas y Oficios Artísticos de Zaragoza. Etapa Fundacional (1894-1910), Lección inaugural del curso académico 1985-86, Zaragoza, 1985, pp. 7-8. 
a celebrarse en la ciudad hispalense, construyó uno de los edificios de esa exposición de tal modo que, pasado el certamen, sirviera para la instalación de la propia Escuela ${ }^{55}$. Por su parte, el discurso recogido en la memoria del curso 1928-29 de la Escuela de Artes y Oficios de Málaga, empieza diciendo: El curso 1928 a 1929 será señalado en la historia de la Escuela de Artes y Oficios Artísticos de Málaga, por el esfuerzo que supone la concurrencia a la Exposición Ibero-Americana de Sevilla y el éxito obtenido alli56.

No es sorprendente, pues, que la Escuela de Artes y Oficios de Vigo tuviera un papel destacado en la Exposición de Arte Gallego de 1924, celebrada, precisamente, en el local de la propia Escuela ${ }^{57}$. Tampoco lo es que al II Certamen Regional del Trabajo de 1935, celebrado en Ferrol, acudieran todas las Escuelas de este género de Galicia, así como las Escuelas del Trabajo de Coruña, Pontevedra, Vigo y Ferrol. Lo que allí se exhibe, escribe el Secretario de la Escuela de Artes y Oficios de Vigo, Edmundo Novoa, que acompañó a los escolares del centro a dicha exposición, son los productos de nuestra tierra y el esfuerzo de nuestros artistas e intelectuales ${ }^{58}$. En este certamen se incluía una Sección denominada, precisamente, "Escuelas de Trabajo y de Artes y Oficios". La Escuela de Artes y Oficios Artísticos de Santiago consigue allí un Diploma de Honor por el conjunto de sus trabajos.

Las Exposiciones (universales, internacionales, nacionales, regionales...) sirvieron a las Escuelas de Artes y Oficios para múltiples fines: dar a conocer las obras de sus profesores y alumnos, estudiar las nuevas tendencias del arte y la industria, aprender las diversas metodologías de la enseñanza de estas disciplinas, entrar en contacto con las innovaciones industriales y científicas, familiarizarse con las técnicas del arte y de la industria, adquirir una visión globalizada del mundo del arte y la ciencia, propiciar unas serie de actividades didácticas encaminadas a la consecución de diferentes objetivos pedagógicos, poner de manifiesto la relación entre arte e industria, etc.

En las exposiciones se ponía de manifiesto el nivel industrial alcanzado por países y regiones, así como las lagunas de otros. Hemos visto esto en el ejemplo de la Exposición Universal de Londres de 1851, y lo mismo podríamos decir de las de 1862 y 1867 con respecto a Francia. Inglaterra, la patria de los grandes inventos industriales, y que se creía en posesión del cetro de la industria, lo vio vacilar en la Exposición de 1851 (....) Francia, a su vez, empezó a entrever en la Exposición universal de 1862, y pudo comprobar en la de 1867, por el órgano de su Jurado, que la industria nacional, de tan merecido renombre por

55 Escuela de Artes y Oficios Artísticos y Bellas Artes de Sevilla. Memoria del curso 1917-18, Sevilla, Imprenta y Librería de Eulogio de las Heras, Sierpes, 13, 1918, p. 5. En efecto, en 1933 la Escuela se instala en la Plaza de España, "en un local espacioso y gran subvención estatal" (Escuela de Artes y Oficios Artísticos y Bellas Artes de Sevilla. Memoria del curso 1938-39, Sevilla, Imprenta y Librería de E. de las Heras, Sierpes, 13, p. 5).

56 Escuela de Artes y Oficios de Málaga. Memoria de 1928-29.

57 Exposición cuyo principal gestor, Herberto Blanco Rodríguez, era profesor y secretario del centro, y en la que participaron Carlos Sobrino, como expositor, y los profesores de la Escuela Alejandro Curty, Maximiliano Vidales y Ventura Requejo, en las vocalías (G. González Martín: "Reválida de galleguidad. La Exposición de Arte de 1924 en Vigo", en Castrelos, 7-8 (1994-95), revista del Museo Municipal "Quiñones de León” de Vigo, pp. 221 a 241. 58 Archivo de la Escuela de Artes y Oficios de Vigo. Edmundo Novoa, "Memoria de la excursión a El Ferrol, julio de 1935". 
su tradicional buen gusto, se había estancado, y aún degeneraba, debido a las deficiencias del aprendizaje en práctica ${ }^{59}$. Redunda en la idea el mismo Díaz de Rábago en el discurso inaugural de 1891 manifestando que se hizo patente en las Exposiciones universales de 1851 y 1867, la falta de cultura industrial y artística de los operarios de Inglaterra y de Francia; las cuales, reaccionando, y para ocurrir al remedio, instituyeron o desarrollaron enseguida variadas escuelas de enseñanza técnica, entre ellas la llamada de aprendices ${ }^{60}$.

Estas muestras sirvieron también, como señalamos más arriba, para poner de manifiesto la relación entre arte e industria - más concretamente la influencia del arte en la industria - tema de constante actualidad en todo el periodo estudiado. Según Juan José Benito, el declarar libre en España, ya en 1785, el ejercicio de las bellas artes, antes vedado a los plebeyos, fue un paso de gran trascendencia y el reconocimiento explícito de que sin las bellas artes no pueden crecer ni mejorarse las artes mecánicas ${ }^{61}$. El citado Díaz de Rábago afirmaba en 1888 que en la enseñanza del Dibujo y del Modelado y Vaciado se habrá de marcar la diferencia, derivada de la misma índole de las cosas, que media entre las Escuelas de Artes y Oficios y las de Bellas Artes, pues dado que según el precepto de Horacio debe considerarse como dechado la alianza de lo útil con lo bello, cada una de ambas instituciones tiene a él por distinta senda, cultivando un fin propio como principal, al que subordina el de la otra: el industrial (utile) las primeras, el artístico o estético (dulce) las segundas ${ }^{62}$. Y Ramón Núñez Fernández, profesor de Modelado y Vaciado de la Escuela de Artes y Oficios de Santiago, no duda en afirmar, en su discurso de 1897:

La injustificada demarcación que ha pretendido seguirse haciendo entre la industria y el arte, queda bien probada al tropezar con las dificultades que lleva consigo el incluir entre los que han logrado hacerse célebres como artistas de reconocido genio a Ghilberti, simple fundidor de bronce; a los Arfes y Benvenuto, plateros; a Bernardo Palissi, modestísimo alfarero; al gran esmaltador Penicot, a los vidrieros Juan de Valdivieso y de Santillana, a varios rejeros, sin fin, a hombres por cuyo manual oficio tan sólo serían considerados como artífices y que por las aplicaciones de sus conocimientos artísticos a sus respectivos oficios tenemos y debemos de reconocer como verdaderos artistas. El sorprendente éxito que la industria francesa obtuvo sucesivamente en las exposiciones de París de 1855 y 1876, vino a demostrar que el arte es el alma, es la condición más esencial de la industria.... ${ }^{63}$

59 Archivo de la Escuela de Artes y Oficios Artísticos de Santiago de Compostela. Joaquín Díaz de Rábago, Delegado regio, Director de la Escuela: Solemne inauguración de la Escuela de Artes y Oficios de la ciudad de Santiago verificada el domingo 19 de febrero de 1888, Santiago, Escuela Tipográfica del Hospicio, Asilo de S. Agustín, 1888, pp. 15-16.

60 Ibídem, Sesión Solemne de inauguración del curso de 1891-92, Santiago, Imprenta de José M. Paredes, Virgen de la Cerca, 30, 1891, p. 59.

61 Juan José Benito: Las Escuelas Estatales de Artes y Oficios y La Educación del Obrero en España (18711900), Madrid, Villena Artes Gráficas S. A., 2002, p. 193.

62 Archivo de la Escuela de Artes y Oficios Artísticos de Santiago de Compostela. Joaquín Díaz de Rábago, Delegado regio, Director de la Escuela: op. cit., p. 22.

63 Archivo de la Escuela de Artes y Oficios Artísticos de Santiago de Compostela. Solemne Distribución de Premios a los alumnos del Curso de 1896-97, verificada en la sesión de 1o de Octubre de 1897, Santiago, Imprenta de José M. Paredes, Virgen de la Cerca, 30, 1897, pp. 8-9. 
Por su parte, en el discurso inaugural de 1896-97 de la Escuela de Artes y Oficios de Vigo se establece sin ambages que el arte sigue paso a paso la dirección y marcha progresiva de la ciencia ${ }^{64}$. Todavía en 1932 se hacía preciso dejar claras estas cuestiones, como lo demuestra el discurso del director de la Escuela de Artes y Oficios de Coruña de ese año, quien asegura que es el Arte consciente, labrado, una de las manifestaciones de la ciencia misma. En efecto: las leyes de la perspectiva se fundan en la Geometría así como ésta, a su vez, se basa en otras disciplinas del saber humano. La anatomía, la euritmia, el ritmo, la técnica de cada Arte, el conocimiento de materiales, etc., etc. ¿Qué son sino ciencia?65.

Señalemos también que, en ciertos casos, las exposiciones servían también para conocer nuevos métodos de enseñanza. Citemos como ejemplo el del II Certamen Regional del Trabajo de Ferrol, de 1935, en el cual la Escuela de Artes y Oficios de Vigo exhibió una metodología para este tipo de enseñanzas, tal como leemos en la memoria del alumno M. R. Novelle, cuando describe lo expuesto en dicho Certamen: De Dn Edmundo Novoa se hallaban unos trabajos relacionados sobre la forma de enseñanza en las Escuelas.... ${ }^{66}$

\section{Las exposiciones como recurso didáctico de las Escuelas de Artes y Oficios}

Las exposiciones constituían una extraordinaria fuente de actividades didácticas tendentes a lograr diversos objetivos educativos: funcionales, instrumentales, formativos, básicos, complementarios, etc. Al ir las exposiciones asociadas a las excursiones, estos objetivos se diversificaban dando lugar a todo un conjunto metodológico que dejaba ver la influencia de la I. L. E. Pues la Institución influyó en escuelas de artes y oficios como la de $V_{i g}{ }^{67}$, sobre todo, en los aspectos metodológicos. Esta influencia se deja sentir más claramente en la Escuela viguesa tras la llegada al centro - primero como profesor de Mecánica Aplicada y luego, también, como director - del institucionista Fernando García Arenal, hijo de la insigne penalista Concepción Arenal ${ }^{68}$. Aspectos como el aprendizaje basado en la experiencia del taller, las visitas prácticas a fábricas y talleres, las excursiones instructivas o prácticas, la finalidad práctica, o el aprendizaje sensorial, serán una constante del centro en todo el primer tercio del siglo XX. En la época republicana se introduce la coeducación, y las alumnas acuden ya a las excursiones junto con sus compañeros.

Las exposiciones, tomadas en conjunto con las excursiones para acudir a las mismas, eran, pues, un extraordinario medio educativo que propiciaba una serie de actividades que

64 Archivo de la Escuela de Artes y Oficios de Vigo. Discurso inaugural y Memoria de Secretaría leídos en la apertura del curso académico de 1896-97, Vigo, Imprenta de La Concordia, 1896, p. 7.

65 Escuela de Artes y Oficios de La Coruña. Memoria reglamentaria. 1932, p. 8.

66 Archivo de la Escuela de Artes y Oficios de Vigo. Manuel R. Novelle: Excursión organizada por la Escuela de Artes y Oficios de Vigo a diversos puntos de la región gallega. Padrón, Santiago, Coruña y Ferrol. Carpeta de Memorias de Alumnos, p. 9.

67 Véase la obra de A. S. Porto Ucha: La Institución Libre de Enseñanza en Galicia, Sada (A Coruña), Edicións do Castro, 1986.

68 Archivo de la Escuela de Artes y Oficios de Vigo. Diversos documentos. 
servían para alcanzar diversos objetivos educativos. Estos objetivos podrían clasificarse del siguiente modo:

- Objetivos sociales: intercambio de experiencias, conocimiento del medio, fomento del respeto mutuo, desarrollo de la empatía, colaboración, aprecio a otras culturas...

- Objetivos lingüísticos: desarrollo y perfeccionamiento de la expresión oral y escrita $^{69}$, ordenación de las ideas, incremento de vocabulario, formación del espíritu crítico, desarrollo de la comprensión oral, conocimiento de otras lenguas, perfeccionamiento de la caligrafía y/mecanografía...

- Objetivos cognitivos: desarrollo de las capacidades cognitivas, desarrollo sensorial, desarrollo de la capacidad de observación, aprender a tomar notas, desarrollo de la memoria, adquisición de nociones geográficas, históricas, científicas, artísticas, comerciales, laborales, etc.; identificación de instituciones, máquinas, aparatos, etc.; formulación y resolución de problemas, adquisición de habilidades, adquisición de diversas técnicas, conocimiento de obras y técnicas científicas, artísticas y comerciales, sistematización, generalización, análisis, síntesis, deducción, inducción...

- Objetivos artísticos: definición y desarrollo del gusto y de la capacidad de apreciar la belleza, fomento de la creatividad artística, identificación de obras de arte, artistas, corrientes artísticas, etc.; perfeccionamiento del dibujo, la pintura, el diseño, el modelado, etc.; diferenciación de aspectos y detalles...

- Objetivos de actitud, éticos o morales: desarrollo del amor al aprendizaje, fomento del aprecio al trabajo, fomento de la equidad, creación de hábitos de cooperación, fomento de la amistas, aprecio a valores como el esfuerzo, el tesón, la humildad, la generosidad; desarrollo de la autoestima, desarrollo del espíritu crítico...

- Objetivos físicos: desarrollo sensorial y de las capacidades físicas, fomento de prácticas deportivas como la marcha, la natación, etc.

Las actividades que llevaban a la consecución de estos objetivos estaban contenidas en los tres grandes momentos: la preparación de la excursión y exposición, la realización de la excursión y visita a la exposición, y la realización de la memoria individual y preceptiva que los alumnos debían presentar a la vuelta.

La preparación previa, tras la selección de alumnos, incluía la presentación, por parte de un profesor o profesores, de la excursión, su lugar, el itinerario a seguir, el transporte, la índole de la exposición, algunas nociones previas a lo que allí se vería, etc. Si la Escuela participaba en la exposición con pabellón propio, los alumnos participaban también en esa fase de preparación ayudando a trasladar materiales y objetos, y a montar dicho pabellón, como fue el caso de la Exposición Regional de 1909 de Santiago de Compostela. Los

69 En todo el periodo estudiado, no hemos hallado pruebas de que en las Escuelas de Artes y Oficios de Galicia se hubiese utilizado la lengua Gallega como lengua instrumental en las clases. 
objetivos a alcanzar serían los de desarrollo de la capacidad de cooperación, respeto mutuo, etc., Estaba también el requerimiento a los alumnos, por parte de profesores y dirección, de una memoria escrita que éstos deberían presentar obligatoriamente en los días inmediatos al regreso. Se les prevenía de que debían tomar nota "in situ" de los datos que necesitarían para la redacción de ese trabajo, lo que desarrollaría su capacidad de observación, perfeccionaría su expresión escrita, fomentaría sus capacidades de síntesis, análisis, etc. Estas notas eran tomadas, efectivamente, por los escolares, a lo largo de la excursión y frente a las exposiciones, tal como se aprecia en diversas memorias de alumnos de la Escuela de Artes y Oficios de Vigo que se conservan en el archivo de este centro.

El viaje en sí variaba según el lugar al que se dirigiesen y los medios con que se contase. Podía ser a pie, o en tranvía o automóvil, si se trataba de la propia ciudad o sus alrededores; y podía ser en autocar o en tren si el destino era más lejano. Pero siempre había lugar a marchas y caminatas (lo que servía para alcanzar objetivos físicos), no sólo para visitar la propia exposición sino también para acudir, de paso, a visitar fábricas, centrales hidroeléctricas, instituciones, otras Escuelas de Artes y Oficios, lugares de interés cultural 0 artístico, etc. Los alumnos de la Escuela de Artes y Oficios de Vigo se trasladaban a los lugares cercanos en ómnibus, así como a diferentes puntos de Galicia y a ciudades del norte de Portugal, y se desplazaron en tren a la Exposición Universal de París de 1900 y a la Exposición Universal de Barcelona de 1929.

Tomando como ejemplo esta última, excursión que tuvo lugar entre los días 8 y 18 de diciembre de 1929, ambas fechas incluidas, he aquí los lugares que los escolares visitaron en la ciudad condal, en compañía de uno de sus profesores y un guía de la Casa Marsans: los pabellones de la propia Exposición, la alcaldía, el Palacio de Justicia, la Diputación, la Sociedad Alpinista de Cataluña, un taller de Cierres Metálicos, la Aeronáutica Naval, la catedral de la Sagrada Familia, el Tibidabo, y la Escuela de Artes y Oficios de la ciudad ${ }^{70}$. A lo largo de estas visitas se llevaban a cabo una serie de actividades que llevaban a alcanzar todos los objetivos que hemos expuesto.

Las memorias preceptivas del viaje y de la visita a la exposición debían ser presentadas a la dirección de la Escuela en los días siguientes al regreso, y podían ser realizadas a mano 0 a máquina (si se trataba de alumnos que asistían a la clase de Mecanografía), y a menudo incluían dibujos de máquinas o aparatos observados en las exposiciones o talleres visitados. En todo ese proceso de realización y presentación de la memoria, el alumno debía desarrollar múltiples capacidades y perfeccionar diversas habilidades. Analizando algunas de las conservadas en el archivo de la citada Escuela de Vigo podemos observar en qué medida podían ser alcanzados los objetivos propuestos.

Una muestra de la habilidad descriptiva nos la ofrece la alumna M. Teresa V. González en su memoria del viaje a Ferrol, para visitar el II Certamen Regional del Trabajo:

En la mañana del día 17 de julio de 1935, reunidos junto a la Escuela de Artes y Oficios de

70 Archivo de la Escuela de Artes y Oficios de Vigo. "Comunicado de los vocales de la Junta Directiva designados por ésta para organizar la excursión a Barcelona, 2-12-29”, Carpeta de Excursiones. 
Vigo, los excursionistas, alumnos de este centro, esperamos llenos de alegría e inquietud la hora de emprender el viaje.

Llega, por fin, el tan esperado momento, y el Sr. Secretario llama a lista a los jóvenes que hemos de realizar tan magnífica excursión. Una vez acomodados en nuestros sitios, comienzan a andar los dos automóviles y poco a poco vamos dejando el centro de la ciudad. Son próximamente las siete y media del día y como el sol está claro, el mar apacible y el cielo azul, el paisaje que se ofrece a nuestra vista es preciosísimo. Vamos dejando Vigo, bullicioso y comercial, y se presenta en el tranquilo mar el Lazareto, en la ensenada de San Simón donde fueron hundidos, en 1702, los galeones que venían cargados de oro procedente de América. Penetramos en Redondela, importante por su estación de ferrocarril, y pasamos por medio del mercado, continuando después entre verdes campiñas y pinares, viendo de vez en cuando alguna pequeña casita con niños a la puerta que nos miran con curiosidad $(\ldots)^{71}$

La capacidad de observación y el dominio léxico pueden apreciarse en el siguiente fragmento de la memoria del alumno J. Pol sobre la Exposición Universal de Barcelona de 1929, describiendo el parque de la exposición:

Al final de la avenida, surgiendo de la base del Palacio Nacional, un torrente de agua se despeña inundando de claridad rojo-violeta todo cuanto a él rodea. Son las cuatro cascadas de 2.500 litros de agua por segundo; las más caudalosas que, no la naturaleza, sino la mano del hombre crea en el mundo (...) Los palacios de Alfonso XIII y Victoria Eugenia muestran sus fachadas, iluminadas con tonos de oro que luego se convierten en un intenso rojo. En último término rematando esta fantástica perspectiva de ensueño, surge en el obscuro (sic) fondo del cielo la soberbia silueta del Palacio Nacional, con la fachada intensamente iluminada en color azul, las torres posteriores de carmín y las cúpulas brillando cual si fueran de materia luminosa. Y para remate de esta visión única la imponente mole del Palacio queda coronada por múltiples haces de luz surgidos de potentísimos reflectores que elevan el eco luminoso del certamen hasta más de 100 kilómetros a la redonda $(. . .)^{72}$

$Y$ el manejo de los datos retenidos en su toma de notas se pone de manifiesto en este otro, del alumno Ismael $\mathrm{H}$. Baamonde, sobre la misma excursión pero, en este caso, la visita al templo de la Sagrada Familia:

Una vez construido, el templo tendrá capacidad para estar cómodamente sentados 14.000 fieles; la puerta denominada "El Paraíso" es una maravilla por su ornato, dos grandes columnas apoyadas sobre enormes tortugas sostienen el dintel de la puerta, sobre dicho dintel la piedra labrada cae en forma de nieve haciendo a la vista del espectador un efecto fantástico: las piedras parece que se van a desprender; unas columnas inclinadas dándonos la sensación de que el Arquitecto estaba loco cuando proyectó eso; hemos subido a una de las torres (la más pequeña) que cuenta con 376 escalones y con una altura sobre el nivel de

71 Archivo de la Escuela de Artes y Oficios de Vigo. M. Teresa V. González: Memoria de un viaje de estudios. Vigo. Padrón. Santiago. Coruña. Ferrol. Santiago. Vigo. Carpeta de Memorias de Alumnos. 1935, 31 páginas. En estos fragmentos de memorias de alumnos han sido respetadas las puntuaciones y la ortografía.

72 Ibídem. J. Pol: Memoria de J. Pol sobre la exposición de Barcelona 1929. Carpeta de Memorias de Alumnos. 
la calle de 112 metros, faltando aún por construir muchas, entre las cuales la mayor tendrá 173 metros de altura; en dicha iglesia hay solamente trabajando 25 obreros y se hace con las limosnas que se entregan para poder visitarla $(. . .)^{73}$

Este mismo alumno, unas líneas más abajo, demuestra de nuevo su capacidad para apreciar la belleza, al hablar de esa exposición:

La Exposición Internacional es algo grande, todo lo que podamos decir o escribir es poco comparado con la realidad; la primera impresión que nos causó cuando la vimos por primera vez, cuando vimos la denominada Fuente Mágica y el alumbrado, no la podemos expresar por medio de estos renglones, nos parecía un cuento de hadas de los que leíamos cuando pequeños (....)

La importancia de los detalles queda patente en el trozo siguiente, perteneciente a la memoria de B. Alonso Costas, sobre la excursión a Ferrol en 1935, para ver el II Certamen Regional del Trabajo, y otros lugares visitados:

Por la mañana hemos visitado el salto de aguas de la Fervenza, en un monte de unos 250 mts de altura y a un desnivel de un $20 \%$ aproximadamente. Hay tres grupos de alternadores que dan una fuerza de 5.550 vatios, 26 amperímetros y una potencia de 150 kilovatios. La corriente que producen estos alternadores pasa a unos elevadores que hay al lado para ser después distribuida $(\ldots . .)^{74}$

Por su parte, Manuel R. Campos describe fielmente, con un mínimo de palabras y perfecta ortografía, la máquina del remolcador Galicia, durante la visita a Ferrol:

La máquina era de triple expansión, esto es de alta, baja y media, con distribución ordinaria (válvula distribuidora). Los cambios de marchas se hacían por una maquinilla. La caldera era de tres hornos y tubular. Tenían además una bomba de vapor auxiliar llamada comúnmente caballito, que tenía por objeto alimentar la caldera, y una máquina conectada a una dinamo para producir corriente eléctrica para todo el barco. El remolcador y la máquina eran holandeses $(\ldots . .)^{75}$

La visita al salto de Fervenza sirve a C. Boullosa López para realizar un ejercicio matemático aplicando las fórmulas aprendidas en las clases:

Potencia de los alternadores: Voltios $=5550$ Amperes $=270$ Coseno de ángulo de fase $=0,8$

La potencia en kilovatios: $\mathrm{W}=\mathrm{V} \times \mathrm{I}, \mathrm{W}=5550 \times 270=1500000 \mathrm{KW}=1.500$ Revoluciones $=$ 750 Frecuencia $=50$ periodos por segundo $(\ldots . .)^{76}$

73 Ibídem. Ismael H. Baamonde: Memoria de Ismael H. Baamonde sobre la exposición de Barcelona de 1929. Carpeta de Memorias de Alumnos.

74 Ibídem, B. Alonso Costas: Memoria de la excursión de los alumnos de la Escuela de Artes y Oficios de Vigo al Certamen del trabajo de Ferrol. Presentada por el alumno B. Alonso Costas. Carpeta de Memorias de Alumnos, 11 páginas.

75 Ibídem. Manuel R. Campos: Memoria de la excursión a Ferrol celebrada por alumnos de la Escuela de Artes y Oficios de Vigo. Carpeta de Memorias de Alumnos, 15 páginas.

76 Ibídem. C. Boullosa López: Memoria de la excursión realizada por los alumnos de la Escuela de Artes y Oficios de Vigo los días 17, 18, 19 y 20 de Julio de 1935. Carpeta de Memorias de Alumnos, 13 páginas. 
La alumna Eulalia Gómez observa los aspectos metodológicos de la Escuela Profesional del Trabajo de la ciudad departamental:

En este Centro pudimos apreciar por una pequeña exposición que allí está instalada, cómo se va inculcando a los alumnos de la menor edad el arte y el trabajo. Desde casi su ingreso, y con las primeras nociones, ya se les enseña a los niños el dibujo y la confección de figuras de cartulina, hasta que se van perfeccionando y adquiriendo conocimientos elementales para pasar a la enseñanza superior de cada asignatura. Es interesantísimo el método de enseñanza seguido en esta Escuela, como así los trabajos expuestos $(. . . .)^{77}$

En el siguiente párrafo, de la memoria de J. M. Corral, observamos el juicio crítico del alumno al describir una parte de lo expuesto en el II Certamen Regional del Trabajo:

Está dividido en secciones. Se observa una rigurosa selección y mucho gusto en el acondicionamiento. A la derecha, entrando, se encuentra el stand expositivo de Labores y otros trabajos femeninos. Ocupa toda la sala. La Escuela Elemental de Ferrol presenta preciosos bordados, puntillas, cojines y otras labores de la mujer, ejecutadas con esmero y limpieza. La Escuela Normal de Lugo presenta también un gran estock (sic) de trabajos femeninos. Destacan un artístico chinero en cartulina fina, en miniatura, de irreprochable ejecución y de un gran efecto visual; le sigue en méritos un pazo gallego; luego un molino de viento, unos jarrones de estilo griego, muy bien construidos. Sigue en méritos la extensa colección de trabajos que presenta la Escuela de Artes y Oficios viguesa. Lo que más destaca de nuestra sección son unos sombreros construidos en papel, una bandeja y dos cojines bordados, artísticamente trabajados. Hay más trabajos femeninos, pero de escaso mérito ${ }^{78}$.

El mismo escolar demuestra también, además de un dominio de los estilos artísticos (adquirido, sin duda, en las clases), un cierto conocimiento de los personajes gallegos de la época que no parece ser sólo fruto de unas indicaciones de profesores o guías:

En cerámica nos llaman la atención algunas ánforas de estilo romano, griego, macedónico y de Sèvres, y un cuadro representando una rincón huertano en el que aparecen plácidamente pescando unos y recreados en tierra otros, varios frailes dominicos (....) En la sección siguiente vemos dos siluetas con las efigies de Valle Inclán y Basilio Álvarez, que acaparan la atención del público. Están ejecutados por el gran artista gallego Díaz Baliño (....) Rivero, el genial tallista gallego, presenta un colosal oso hormiguero, los famosos "comuneros" en el cadalso y una escena selvática, que un primor de ejecución $(. . .)^{79}$

En las Factorías de la Constructora Naval demuestra también la capacidad de observación de los detalles y su poder de retención (teniendo en cuenta que se trata de un alumno que no es mecánico sino litógrafo), cuando describe el crucero Canarias:

77 Ibídem. Eulalia Gómez: Memoria de la Excursión escolar-instructiva realizada por los alumnos de la Escuela de Artes y Oficios de Vigo a diversas localidades de Galicia. Julio de 1935. Carpeta de Memorias de Alumnos, 5 páginas.

78 Ibídem. J. M. Corral: Julio del año 1935. Memoria de un viaje de estudios por la región. Carpeta de Memorias de Alumnos, 21 páginas.

79 lbidem. 
A su derecha se halla en construcción también su gemelo Baleares, y a la izquierda el acorazado España, en reparación. Un poco más allá se están dando los últimos toques a los barcos de guerra Guanajuato y Querétaro, para la marina mejicana. El Canarias con su gemelo Baleares, son de los cruceros más potentes del mundo. Están movidos a petróleo. La eslora de estos buques desde el codaste a la roda, sobre cubierta, es de 190 metros. Sus torres de proa y castillos son de grueso espesor. Los cañones, desde el interior de sus torres, son movidos eléctricamente en todas direcciones. Y para impedir que los cañones bajen más de lo normal, existe la llamada arboladura de obstáculos. Los proyectiles son subidos a las torres por unos ascensores interiores. Tiene este destructor un verdadero porte elegante $(\ldots . .)^{80}$

En tanto que su compañero, el ya citado Manuel R. Novelle, demuestra el conocimiento de los acontecimientos de aquellos días cuando señala que en el castillo de San Felipe, en la ría ferrolana, se hallan algunos detenidos de cuando fue el pasado movimiento revolucionario de Asturias, para añadir, al hablar del Depósito de Armas del Arsenal Militar, que había allí, entre otras, un número bastante elevado de armas que transportaba el "Turquesa" para el pasado movimiento revolucionario, siendo el único alumno del grupo que menciona estos datos ${ }^{81}$.

\section{La selección de alumnos}

La selección de alumnos y alumnas corría por cuenta del centro. Se seleccionaba a los alumnos de cada sección o rama de los estudios siguiendo criterios de aplicación, edad y aprovechamiento. En un principio, esta selección no fue muy estricta, lo que fue corrigiéndose año tras año. En general, se trataba de los alumnos que terminan sus estudios y que más se han distinguido por su aplicación ${ }^{82}$. Para ir a la Exposición Universal de Barcelona de 1929, por ejemplo, fueron seleccionados, por la Escuela de Artes y Oficios de Vigo, siete alumnos, que han sido elegidos entre los que, reuniendo mayores méritos en las calificaciones obtenidas y asistieron al mayor número de clases, asistieron a ellas con más asiduidad y son mayores de 19 años. Todos son muchachos formales, que aceptaron la designación con extraordinaria alegría, y sabrán aprovechar, seguramente, las enseñanzas que la excursión proporcione ${ }^{83}$. Estas normas se establecieron formalmente en la sesión de la Junta Directiva de 31 de julio de 1934, siendo aprobadas por el Ayuntamiento el 24 de agosto de ese $a \tilde{n} 0^{84}$. Los criterios de selección eran similares a los de otras Escuelas del género. El número de alumnos y alumnas (recordemos que hasta los años republicanos no van las alumnas a estos viajes, al menos en la Escuela de Vigo) que eran elegidos por el centro para acudir a estos certámenes, era siempre reducido, debido a imperativos

80 Ibidem.

81 Ibídem. Manuel R. Novelle, op. cit.

82 Archivo de la Escuela de Artes y Oficios de Vigo. "Comunicado de E. Novoa a la Cámara de Comercio 156-1934".

83 Archivo de la Escuela de Artes y Oficios de Vigo. "Comunicado de los vocales de la Junta Directiva designados por esta para organizar la excursión a Barcelona, 2-12-1929". Carpeta de Excursiones.

84 Archivo Municipal de Vigo. Escuela de Artes y Oficios. Legajo 43. Año 1934. La Escuela de Artes y Oficios de Vigo nació por iniciativa privada en 1886, pero se haría municipal dos años más tarde, en 1888. 
económicos, lo cual constituye un aspecto que, en ocasiones, fue objeto de crítica. A la Exposición de París de 1900 fueron enviados seis alumnos de un total aproximado de 400 alumnos varones matriculados ese año; a la Exposición Universal de Barcelona de 1929 fueron enviados 7, de un total aproximado de 700 alumnos varones matriculados ese año; y al II Certamen del Trabajo, de Ferrol, de 1935, fueron enviados 50 alumnos y alumnas de un total aproximado de 900 alumnos y alumnas matriculados ese año. Naturalmente, sólo los de los últimos cursos tenían opciones para ser seleccionados, lo que hace que la proporción sea menor. Pero estas cifras muestran con bastante claridad que lo reducido del número de alumnos seleccionados para asistir a estas muestras resta eficacia a sus resultados positivos en la labor educativa del centro, aunque no por ello son despreciables.

\section{Conclusiones}

La relación entre las Escuelas de Artes y Oficios y las Exposiciones fue constante desde el comienzo de unas y otras - casi a la vez - hasta finales de los años 30 del pasado siglo. Si nos ceñimos a las Escuelas de Galicia, esta relación se concreta en numerosas participaciones de profesores y alumnos en dichas muestras, con la presentación de obras de arte - pintura, escultura, orfebrería, etc. - realizaciones de tipo industrial, y hasta métodos de enseñanza, y con la asistencia a las mismas de grupos de alumnos seleccionados por las Escuelas y acompañados de profesores y otros miembros del personal de los centros. Asistencia sufragada por estas Escuelas (del fondo de presupuestos destinados a tal efecto), junto con ayudas exteriores que iban desde becas estatales hasta aportaciones privadas.

Estos viajes constituían por sí mismos excursiones escolares, prácticas e instructivas. La combinación de excursión y asistencia a las Exposiciones propiciaba una serie de actividades didácticas tendentes a la consecución de diversos objetivos educativos, programados o no, constituyendo un aspecto metodológico de estos centros de educación técnica popular de adultos característico de esos años. Aspecto metodológico de los menos investigados hasta la fecha pero que, según lo que se desprende del estudio de uno de ellos - la Escuela de Artes y Oficios de Vigo -, produjo efectos positivos importantes en la educación de los alumnos, tanto los que asistieron a los certámenes como los que no lo hacian. Los frutos de esta combinación de excursión y exposición se pusieron de manifiesto en la modernización del currículo escolar, de los programas y los planes de estudios, y la metodología; en la formación de los propios escolares enviados a las exposiciones (traducida en una mejora de las notas), en la divulgación de los trabajos del centro, y en el incremento del prestigio del mismo. Si bien los resultados educativos o beneficios de estas actividades se reducen considerablemente debido al escaso número de alumnos seleccionados para asistir a estos eventos.

El interés de las Escuelas de Artes y Oficios por las Exposiciones Universales y Regionales se inscribe en un tiempo en que estas muestras gozaban de gran prestigio (especialmente en los campos industrial y artístico), habiéndose generalizado en la clase obrera la convicción de que la asistencia a las mismas paliaría en parte su atraso. La legislación 
escolar recogió este sentir general y las incluyó en su articulado, así como los reglamentos de los distintos centros, con presupuestos, becas y ayudas para acudir con una representación de alumnos y profesores a estas muestras. Las Escuelas de Artes y Oficios de toda España, en general, y de Galicia en particular, participaron a menudo en las Universales celebradas fuera de España, pero, sobre todo, no dejaron de hacerlo cuando la muestra se celebraba en la comarca donde se hallaba la Escuela.

Este breve estudio ha intentado demostrar de qué manera eran utilizadas las Exposiciones para conseguir ciertos objetivos educativos por parte de estos centros, en la época de desarrollo industrial de Europa en general y de Galicia en particular, tratando de probar también la relación constante entre este tipo de instituciones y las ciencias, la industria, el comercio, el arte y el mundo laboral. 